\title{
Appel à contribution sur les finances publiques citoyennes
}

\begin{abstract}
a confiance des citoyens dans les institutions Let la démocratie semble ébranlée. Les questions financières n'échappent pas à cette crise de légitimité, elles en sont sinon le cœur, du moins le catalyseur. II apparaît alors impératif d'interroger le rapport entre la citoyenneté et les finances publiques, d'observer l'écart réel ou supposé entre la théorie et la pratique.

Si l'on doit saluer un certain nombre d'initiatives de la communauté scientifique sur ces sujets ${ }^{13}$, I'actualité commande d'aller plus loin et de proposer une nouvelle approche/méthode.
\end{abstract}

La Société française de finances publiques (SFFP), qui rassemble la plupart des experts du sujet, et Gestion \& Finances publiques, la revue de référence des professionnels des finances publiques, unissent leurs efforts pour promouvoir des initiatives innovantes qui interrogent le rapport étroit entre finances publiques et citoyenneté. Cette démarche d'ouverture s'inscrit dans une logique réciproque : il s'agit non seulement de rendre accessibles aux citoyens les éléments fondamentaux des finances publiques, mais aussi d'ouvrir la réflexion des experts des finances publiques aux préoccupations et interrogations des citoyens.

Cette nouvelle approche permettrait d'analyser des thèmes de recherche variés et prospectifs comme (la liste n'est pas exhaustive) :

- le renforcement du contrôle démocratique sur les budgets à différentes échelles,

- les budgets participatifs,

- l'ouverture et l'accessibilité des informations budgétaires et comptables aux citoyens,

- l'information et la pédagogie en matière de fiscalité pour améliorer le civisme fiscal,

- les finalités des dépenses publiques,

- le processus de décision en matière financière,

- le consentement de l'impôt.
Sur le ou les sujets choisis, les experts de la SFFP proposeront une analyse de l'état du droit et pourront formuler des propositions concrètes permettant de renforcer l'aspect citoyen des finances publiques. Celles-ci seraient publiées par G\&FP et mises en débat sur les sites de chacune des organisations. Ce partenariat entre experts a vocation à être complété par un ou plusieurs partenaires qui pourraient relayer les sujets auprès du grand public.

Le partenariat pourra porter :

1 / sur la promotion de thèmes particuliers sur lesquels les partenaires produiraient des données, des études, des articles avant, le cas échéant, de rédiger une proposition formelle à l'attention des pouvoirs publics

2/ sur une stimulation de la recherche et une valorisation des initiatives concrètes relatives aux finances publiques citoyennes (prix, publications...) $3 /$ sur un service d'accompagnement d'initiatives : des experts et des praticiens, mis en relation avec les porteurs de projets, répondraient à leurs questions...

Ces différents modalités, et d'autres (colloques...), peuvent être proposées simultanément ou successivement.

Nous attendons vos suggestions de thèmes de réflexion et d'action, vos propositions d'articles, vos indications $d$ 'initiatives à soutenir.

\section{Audrey Rosa}

Maître de conférences à I'Université de Lille (Centre Droit et perspectives du Droit EA n ${ }^{\circ}$ 4487) audrey.rosa@univ-lille.fr

\section{Michel Le Clainche}

Rédacteur en chef de Gestion \& Finances publiques leclainchemichel@gmail.com 